Giving and Receiving Care: An Evolving and Enduring Relation

\author{
Philip Y. Kao \\ Ph.D. Candidate \\ University of St. Andrews \\ Philip.Y.Kao@gmail.com
}

Much has been written about the practice of caregiving and care for the elderly, especially in places like the traditional nursing home (e.g. Diamond 1986, Savishinsky 1991, Stafford 2003, Gass 2004). In the United States caregivers commonly refer to those professionals that constitute a cadre of nurses, nursing assistants (both certified and uncertified), therapists, palliative care/ hospice workers - and very often - family members, who help the elderly in a range of activities from ADL's (activities of daily living) to providing companionship, and a host of other tasks. Professional caregivers working in long-term care facilities such as nursing homes (skilled nursing facilities) and assisted living homes often struggle to balance the need for personalizing care and doing everything on time, at the expense of treating residents as simply dress-up dolls. CNA's and uncertified caregivers oftentimes look after more people than they can handle, leaving little room for striking up friendly conversation, expressing empathy, and engaging in simple human companionship. Family caregivers struggle in different ways, but are just as hard-pressed for time especially when their loved ones suffer from dementia related conditions (e.g. Alzheimer's) that demand care and attention constantly. These family caregivers are sons, daughters-in-law, and siblings who are undoubtedly frustrated, and have little or no time to attend to their own stress and health issues. Caregiving in the presence of financial, emotional and family stress also adds pressure to the overall situation. Not only are caregivers personally affected, but the caregivers' families and the people around them are affected by the ensuing stress. It is no surprise then that sociologists and gerontologists have focused their research in the past on such topics as communication between caregivers and the recipients of care, disputes arising between family members and caregivers, and ways to become better, more effective caregivers at home and in the community (e.g. Foner 1995, Ward 2008).

What is interesting for anthropology is a consideration of the caregiving relationship between particular people, and the relationship as a structural feature developing out of a wider social, economic, and historical context. To begin with, my interest in aging has to do partly with the industry and practice of caregiving and what this says about our social and emotional linkages to one another. This is not to say that I am interested in how people are (or are not) culturally obligated to take care of the sick or elderly. Rather, it is the intimate and corporeal relationship that unfolds between a caregiver and a care-receiver that illuminates what it means to be independent/ dependent, whole/broken, and social in specific contexts. Caregiving then is never neutral; i.e. it can sometimes stand in as a metaphor for an assertion of (bio)power (cf. Foucault 2007). I once heard a female caregiver in a nursing home confront a nurse about a particular resident. The CNA was trying to convince the nurse that 'her' resident was acting up and needed some medical attention. After the nurse neglected to pursue any follow up, the CNA walked away noting sarcastically, "Well what do I know? After all I am just the caregiver! I am with her Monday through Friday" In other contexts, caregiving can be operationalized to assert a type of labor identification and experience. Caregivers often say to themselves that, "Not everyone can be a caregiver. It takes passion; you have to like taking care of people. Not everyone is cut out that way". In caregiving, the emotional dimension is far more complex than the sheer physical job of helping someone get out of bed 
routinely, brush their teeth, and getting them to various appointments while making sure that they are 'toileted' every two hours. In fact, caregivers themselves have developed a form of dependency as well. I overheard several times that, "If it wasn't for my residents, I would have quit a long time ago". There is a creeping suspicion (on my part) that caregivers need their residents just as much as the residents need them.

As alluded to above the political economy of caregiving is an evolving and complex enterprise featuring aspects of the government, healthcare (reform), insurance, the medical and legal professions, and capitalism. What I found fascinating during the early phase of my fieldwork was the extreme low pay and the prevalence of caregivers who migrated from other countries such as Sudan, Kenya, and the Bosnia. These people, who were being paid just slightly above minimum wage, had the most direct and intimate contact with someone's dear mother, father, and grandparent. They were the front line of care and social interaction with the residents in the nursing home. In most cases, they were the residents' only emotional and social outlet. I began to wonder if post-Fordism and the transition to the service economy had enabled people to render care in an assembly line manner. It was during my fieldwork, working and volunteering at numerous longterm care facilities, that I began asking whether or not it is the resident or rather the caregiver that is ultimately becoming increasingly alienated.

To shift gears a bit, I would like to address how an anthropological investigation of caregiving can be worthwhile. In addition to the policy and applied research associated with gerontology and sociological studies of aging in society, anthropology can offer a unique perspective toward understanding and describing the 'caregiver phenomenon' that other disciplines do not dwell on in quite the same way. Take two social settings: support groups and the family as examples of how an anthropological analysis of caregiving and caregivers can begin to inform the social nature and relational aspects of aging.

Support groups have long been in existence, providing emotional outlets and social networking opportunities for people suffering from HIV / AIDS to Vietnam war veterans looking to 'reassimilate' into society (e.g. Rhine 2009). Support groups have also come into existence for those dealing with loved ones suffering from dementia and Alzheimer's. Several support group associations for family caregivers seeking respite have gained increasing popularity over the years as well. In these "How to Manage Stress as a Caregiver" support groups, relationships between parent and child, husband and wife, brother and sister are transformed slightly to caregiver and care-receiver. Support groups offer strategies for dealing with everyday situations, by providing frameworks for compartmentalizing and diagnosing their loved one's conditions. They teach such strategies as 'creative lying' in order to alleviate 'unnecessary conflicts', especially when short term memory lapses can spiral into uncontrollable repetitions and irrational expectations. Caregivers listen to one another's problems, and shape their own views through the interplay of sympathy and empathy. They are taught by group leaders to 'enter into the reality' of their loved ones-even if this reality is distorted.

Not only do these support group participants share their experiences and personal stories, but by sharing and commenting on them, their own relationships at home are shaped by the support sessions and what they see as constituting appropriate roles and responses for caregivers and 


\section{Student Column}

care-receivers. Therefore, husband and wife, brother and sister relations are transformed through caregiving into relations of caregiver and care-receiver. One also learns from these support groups that family caregivers are hesitant to ask for help from their neighbors and fellow community church members. The tension between independence and dependence not only matters for the elderly, but also comes into being for caregivers. The cultural push and pull between self-reliance, dependence, and being able to afford 'professional' care are issues that rush to the forefront in many support group sessions. Additionally, during some of the sessions caregivers are attributed a unique status; they are not quite victims, but instead find mutual support and define membership through their hardships and 'war stories'. Their health and wellbeing is also a paramount issue; caregivers are told that they must center themselves first before they can reach out and connect with their loved ones. The support group message places emphasis on a particular western bounded notion of a person first, rather than any relationship. Caregiving as a relation with scripted roles, strategies, and proprieties are imbued with and produce interesting notions of personhood, self care, legal capacity, and the function and nature of social relations within the family.

In the second setting which plays out behind closed doors, caregiving by family members in the home brings forth its own set of issues. Siblings are often in conflict over how care is administered, and typically the issue boils down to who will actually 'do the caregiving'. Many family caregivers complain that they are overburdened, and that other siblings who are close by should offer more support and care. Along with this, issues of family inheritance, financial management, power of attorney, and decision making on behalf of an aging parent are highly contested. Turning to the onset of aging societies, children and their parents will spend more time together now as adults (Harper 2004). Caregiving in this regard produces an unsettling role reversal where the 'children' assume parental responsibilities and care over their mothers and fathers. Women are still predominantly the family caregivers, but even this generality is changing as some sons have started moving back into their parents' home to provide care not only for mom and dad, but for some members of the extended family. In this changing situation, old taboos are being replaced by new ones. Washing one's parent and seeing them naked may no longer cause one to blush, but not having enough money or time to provide a caregiver may actually be a larger source of social embarrassment.

Caregiving is a growing and lucrative service industry of professionals on the coattails of America's (over)reliance on anything medical and certifiable. In the near future, caregiving will surely continue to develop, and innovate in anticipation of the aging baby boomers and their market preferences. As a result, and this something that we already see today, families will continue to outsource care. Children's daycare for example gained prominence when both parents had to work. Checking into a long term care facility such as the traditional nursing home or hiring a fulltime caregiver at home can be seen in a similar vein. Family members who are too busy at work, or do not possess the confidence to provide adequate care for their aging loved ones will seek private caregivers outside the immediate nuclear (and extended) family. What is interesting is not the price tag of care necessarily, but that domestic work, which has long been associated with the sexual division of labor, no longer assumes that caregiving for the elderly is automatically part of domestic work. The interaction and relationships born from domestic labor (which is still not accounted for in the national GDP) and caregiving for the aging, is an interesting site for future research and 
investigation.

What emerges from this brief discussion is a social phenomenon that offers a rich opportunity to explore some of the mainstay anthropological ideas/theories such as kinship, personhood, and social relations. In the post-Schneider world of kinship, folk models on the American family are based on the implicit notion that kinship is the network of people who have to care for you when no one else does become problematic (Schneider 1975). Does paying for an outsider to provide care elicit the same kinship ties and familial emotions as someone who provides care for their own family members directly? What happens if the state takes on more of an active role in caregiving, how will this affect kinship? If caregiving is no longer the domain of kinship and domestic labor, then what becomes of the nuclear and extended families, and how do aging persons view families and their kinship relations differently than before? These are questions that anthropology can address for our own societies and cross-culturally in the wider castings of globalization. If anything, anthropology can help to show that caregiving operates in a social context, and that who and how one becomes a caregiver is sometimes meaningful, and sometimes it isn't.

An anthropology of caregivers can seek to address what actually constitutes care in various cultural settings and societies. Is 'care' a set of (performative, monetary, et al) functions which simply arises out of reciprocation and/or a moral ethic that forces us to look after the elderly, and in the ways that we do? In the future, will every aging person be assigned a caregiver; what will happen to caregivers as they become older? What will be their expectations for the care and experiences they will have in old age? These are just a few of the questions regarding caregiving and caregivers that anthropology could seek to address more of in the future.

Philip Kao is a PhD candidate in social anthropology at the University of St. Andrews. He is researching a Continuing Care Retirement Community (CCRC) in the Midwest, focusing on its history and place in the surrounding community. Additionally, he is researching the social relations at the CCRC, with a focus on kinship and personhood.

\section{Works Cited}

Diamond, T.

1986 Making Grey Gold: Narratives of Nursing Home Care. Chicago: University of Chicago Press

Foner, Nancy

1995 Relatives as Trouble: Nursing Home Aides and Patients' Families". In Neil Henderson and Maria D. Vesperi (eds.). The Culture of Long Term Care: Nursing Home Ethnography. London: Bergin and Garvey.

Foucault, Michel

2007 Security, Territory, Population: Lectures at the Collège de France, 1977-78. Graham

Burchell (trans.). Basingstoke, NY: Palgrave Macmillan.

Gass, Thomas Edward

2004 Nobody's Home: Candid Reflections of a Nursing Home Aide. Ithaca: ILR Press.

Harper, Sarah

2004 Families in Aging Societies. New York : Oxford University Press.

Rhine, Kathryn A. 
2009 Support Groups, Marriage, and the Management of Ambiguity Among HIV-Positive

Women in Northern Nigeria. Anthropological Quarterly, 82(2): 369-400.

Savishinsky, Joel

1991 The Ends of Time: Life and Work in a Nursing Home. Westport: Bergin and

Garvey.

Schneider, David M.

1975 The American Kin Universe: A Genealogical Study. Chicago: Dept. of Anthropology,

University of Chicago.

Stafford, Philip (ed.).

2003 Gray Areas: Ethnographic Encounters with Nursing Home Culture. Santa Fe: School of

American Research Press.

Ward, Richard et al

2008 A Different Story: Exploring Patterns of Communication in Residential Dementia Care.

Ageing \& Society, 28: 629-651.

\section{AAGE ON DISPLAY IN NEW ORLEANS 2010}

Things will be a bit different this year. AAA sold all its booths, so we'll be down to basic table and chairs -in or near the exhibit hall. All the usual fun and opportunities will be available:

- Sign up for the AAGE dinner (Friday evening).

- Take a turn at the table-it's a fun way to meet people.

- Buy a T-shirt-our bestseller "Broken Down by Age \& Sex" is good for laughs at the gym! People get them for their mothers too (though some prefer "Aged to Perfection" for mom).

- Renew your membership.

- Display your book - free in return for staffing the booth for two hours.

- Please donate your copy to the silent auction which is raising money for the Jacob (Jay) Climo Student Fund.

- Bid on the books in the Climo Fund silent auction. It's fun, and you might get a good book for a bargain price.

Any questions? Contact Maria Cattell mgcattell@aol.com

Hope to see you there! 